\section{$\underset{\substack{\text { hommes } \\ \text { \& migrations }}}{ }$}

\section{Hommes \& migrations}

Revue française de référence sur les dynamiques

migratoires

$1315 \mid 2016$

Ondes de choc

\title{
Entretien avec Jacques Toubon, le Défenseur des Droits
}

\author{
Marie Poinsot
}

\section{(2) OpenEdition \\ Journals}

Édition électronique

URL : http://journals.openedition.org/hommesmigrations/3718

DOI : 10.4000/hommesmigrations.3718

ISSN : 2262-3353

Éditeur

Musée national de l'histoire de l'immigration

Édition imprimée

Date de publication : 1 juillet 2016

Pagination : 82-87

ISBN : 978-2-919040-36-0

ISSN : $1142-852 X$

Référence électronique

Marie Poinsot, «Entretien avec Jacques Toubon, le Défenseur des Droits », Hommes \& migrations [En ligne], 1315 | 2016, mis en ligne le 01 juillet 2019, consulté le 14 septembre 2020. URL : http:// journals.openedition.org/hommesmigrations/3718 


\section{ENTRETIEN AVEC JACQUES TOUBON, LE DÉFENSEUR DES DROITS}

Réalisé par MARIE POINSOT, responsable du département des éditions du Musée national de l'histoire de l'immigration et rédactrice en chef de la revue Hommes \& Migrations.

Hommes \& Migrations : La revue souhaite faire un état des lieux du travail du Défenseur des Droits. Pouvez-vous nous rappeler les grandes étapes de la prise en compte par l'État français des discriminations? L'impulsion de l'Europe a-t-elle été déterminante ?

Jacques Toubon : Si l'on remonte aux origines, c'est la lutte contre le racisme et la xénophobie, et aussi l'homophobie, qui a introduit ces notions juridiques, en privilégiant l'égalité de droit entre les personnes quelles que soient leurs différences. Ceci remonte à la loi sur la presse de 1881 et puis surtout la loi dite «Pleven », n 72-546 du $1^{\text {er }}$ juillet 1972, relative à la lutte contre le racisme. Mais, pour parler véritablement de la lutte contre les discriminations, il faut se référer aux initiatives prises dans les années 1980 visant à supprimer les règles discriminatoires à l'égard des personnes homosexuelles. Dans la perspective de la progression de l'épidémie du Sida, beaucoup de controverses ont conduit la France à prendre des dispositions pour essayer d'éviter que les porteurs du VIH, les malades, les séropositifs ne soient traités de manière péjorative. Si on met à part cette situation particulière, l'histoire des discriminations est celle de l'introduc- tion dans la culture juridique française de concepts qui étaient au départ anglo-saxons - les droits des minorités, ce qu'on appelle le politiquement correct -, par le truchement de la législation européenne de directives des années 1990-2000 que la France a transposé dans sa propre législation contre les discriminations. Ce faisant, la France a fait évoluer sensiblement sa culture juridique : nous sommes passés d'un principe d'égalité devant la loi à un principe d'égalité par la loi, qui institue les 22 critères de discrimination illégale identifiés dans la grande loi de 2008 sur les discriminations dans l'emploi, les biens et les services, et ses modifications successives. Cette évolution récente a provoqué un véritable bouleversement par rapport à notre culture juridique traditionnelle. Les influences des conventions internationales, par exemple celle sur les droits de l'enfant ou celle sur les personnes handicapées, ont également été déterminantes.

H\&M : Pourquoi est-il important de distinguer les discriminations à raison de l'origine et celles à raison de la nationalité ? Permettentelles de couvrir des populations différentes et des champs d'intervention complémentaires? 
Peut-on également parler de discriminations à raison de l'appartenance religieuse ? Cette distinction implique-t-elle des différences dans les approches et les mécanismes juridiques protecteurs?

J. T. : L'origine et la nationalité font l'objet de traitements juridiques différents. L'origine renvoie principalement à une donnée ethnique réelle ou supposée. Les discriminations à raison de l'origine portent sur la couleur de peau, sur l'accent, sur le patronyme, etc. laissant supposer une origine étrangère. L'origine est un des principaux motifs de discriminations en France, après le handicap et la santé qui comptabilisent à peu près $25 \%$ des cas. Nous les combattons avec beaucoup de vigueur, que ce soit dans l'emploi, l'accès aux biens et aux services. En revanche, la nationalité est une donnée juridique bien balisée dans la mesure où la souveraineté des États les autorise à avoir une police des étrangers (entrée, séjour et éloignement) et, d'autre part, à appliquer des règles différentes aux personnes selon leur nationalité. Un des exemples, qui a toujours été admis, c'est la condition de séjour de dix ans pour l'allocation d'aide aux personnes âgées. Il existe cependant des dispositions relatives à la nationalité qui sont discriminatoires. Un exemple concret : dernièrement, j'ai obtenu, dans la discussion de la loi Égalité et citoyenneté, que l'on réduise considérablement les catégories d'emplois fermés aux étrangers et réservés aux citoyens français. Aujourd'hui se combine, à l'origine, la question de la religion qui ne fait qu'accroître les difficultés et les confusions entre des figures stéréotypées comme «l'arabe » ou « le musulman ». Les discriminations se fondent de plus en plus sur un amalgame de différentes caractéristiques des personnes. Le Défenseur vient de publier un appel à témoignages sur les discriminations selon l'origine dans l'accès à l'emploi qui décrit une situation fort préoccupante à cet égard.

Le genre est bien entendu un autre facteur de discriminations : appartenance sexuelle, orientation sexuelle, auxquelles je souhaite rajouter l'identité sexuelle que la nouvelle loi va heureusement intituler « l'identité de genre ». J'avais publié au cours du mois de juillet une décision-cadre proposant que le changement d'état civil des personnes transidentitaires relève d'une procédure déclarative et non plus juridique. Hélas, tous ces critères de discrimination s'accumulent et se conjuguent. Par exemple, une femme étrangère va devoir affronter d'importantes difficultés dans le domaine de la santé. Nous avons été saisi notamment de cas de femmes étrangères en situation de grossesse non désirée. Certains centres d'orthogénie ne jouent pas toujours le jeu et leur ferment un recours effectif à l'IVG en alléguant la tardivité de leur demande.

$H \& M$ : Les recherches sur ces réalités discriminatoires sont-elles en progression en France? Quels sont les universités et les laboratoires de recherches qui travaillent sur ces problématiques et en étroite relation avec le défenseur des droits ? Le Défenseur des Droits estil en lien avec des programmes de recherche à l'échelle européenne?

J. T. : Le Défenseur conduit et coordonne des travaux d'étude et de recherche en vertu de l'article 34 de la loi organique du 22 mars 2011. Cela fait donc partie intégrante de ses compétences. Le résultat le plus important de cette mission est la grande étude intitulée Trajectoires et origines que nous avons cofinancée. Maintenant qu'elle est enfin publiée, nous allons contribuer à deux exploitations particulières : l'une porte sur le racisme et les discriminations ethniques versus ressources et stratégies par l'Urmis ; l'autre porte sur les sentiments d'injustice et les ressentiments vécus par les descendants d'immigrés à l'école par l'Irev.

Non seulement, le Défenseur a toujours soutenu les travaux de recherche, mais il doit en assurer la promotion, comme en 2013 avec sa participation au programme « Progress » de la Commission européenne, qui nous a permis de publier avec la Cnil le guide Mesurer pour progresser dans l'égalité des chances. Le Défenseur travaille avec des laboratoires 
comme l'Urmis, le Cedis, le Ceri, l'Iscra, Migrinter, l'EHESS. Sur les statistiques, le Défenseur travaille avec la Dares du ministère du Travail, l'Ined et l'Insee, le Service social des migrants, le GIP justice. Au plan européen, il est en lien avec l'Agence des droits fondamentaux, notamment pour l'enquête comparative intitulée «EU Midis ». Cette capacité d'études et de recherches repose notamment sur le triplement du budget études depuis que je suis arrivé et sur la coopération avec des partenaires de plus en plus nombreux. Nous venons d'adresser une grande enquête sur l'accès aux droits et sur les raisons de non-recours au droit auprès d'un panel de 5000 personnes; nous allons l'exploiter avec l'Ined et l'Observatoire des non-recours aux droits (SciencesPo Grenoble), les premiers résultats étant attendus cet automne. Elle mesure les raisons profondes de non-accès aux droits que peuvent être l'origine, la religion, le patronyme, le lieu de résidence.

H\&M : La question n'est donc pas forcément celle des connaissances produites sur les discriminations mais de leur diffusion vers l'opinion par les médias et vers les décideurs politiques ? Le Défenseur a-t-il une mission de diffusion des connaissances dans ce domaine et/ou de sensibilisation et de prévention ? Quelles en sont les actions prioritaires ? Les dispositifs de lutte contre les discriminations sont-ils suffisamment connus et identifiés par les publics ? Le nombre des signalisations, les dépôts de plaintes et les recours ne sont-ils pas proportionnels à la connaissance de ces dispositifs ? Quels sont les autres freins identifiés dans les démarches des victimes de discriminations et que faudrait-il améliorer ?

J. T. : Le premier défi est de faire mieux connaître ces travaux sur les discriminations qui étaient jusque-là souvent tenus sous le boisseau, à l'exception du baromètre établi depuis huit ans avec l'OIT qui, chaque année, suscite une forte médiatisation. Cette année, par exemple, nous avons exploité l'item « apparence physique » et tous les journaux ont commenté les situations des gros, des petits, des grands, etc. dans le monde du travail et le rôle de l'apparence physique dans la discrimination à l'embauche et dans la carrière professionnelle. À travers les rencontres, les colloques et les autres missions, j'essaye de diffuser les connaissances produites par les recherches sur les discriminations. Ainsi, au début de l'année, une rencontre de restitution a permis de diffuser une étude faite avec le ministère du Logement et le CGET sur l'accès au logement social et sur les délais d'attente en fonction des profils des demandeurs. Mais les résultats restent encore insuffisants. D'un autre côté, ces études sont liées aux actions de promotion que le Défenseur des Droits souhaite développer : connaître les causes et les leviers sur lesquels il faut s'appuyer. D'où la publication et la diffusion de guides qui permettent de mesurer et d'accompagner les interventions de prévention ou de lutte contre les discriminations. J'ai mis en place l'année dernière un service de communication qui manquait jusqu'à présent et va piloter toute une série d'actions qui auront pour but d'exploiter le résultat des études à travers des outils d'informations et des ressources.

H\&M : Avec les attentats meurtriers de 2015 et 2016, le contexte français a-t-il évolué ? La question de la lutte contre les discriminations est-elle portée par les médias qui véhiculent aussi des préjugés sur certaines populations? J. T. : Il y a un travail de pédagogie à faire en direction des journalistes pour qu'ils apprennent à déconstruire leur propre discours sur les discriminations. Depuis les attentats de 2015, on observe un climat de sidération dans l'opinion publique qui choisit systématiquement le point de vue sécuritaire et adopte, sur les étrangers et les migrants, le point de vue de la maîtrise des flux partagé par la droite comme par la gauche : il faut mettre davantage de barrières, bloquer les mouvements migratoires à la porte de l'Europe. À partir de là, jusquà cette loi Liberté et citoyenneté dont le titre 3 a pu intégrer, grâce à l'intervention du Défenseur, la 
lutte contre les discriminations, il faut bien reconnaitre que le discours public est devenu largement identitaire depuis dix ans et indifférent à la lutte contre les inégalités. Le discours sur la laïcité est devenu un discours d'exclusion alors que la laïcité devrait, au contraire, mettre l'accent sur la liberté et les libertés. Dans les médias, il est très difficile d’introduire des points de vue comme celui du Défenseur : quand je publie un rapport de 305 pages sur l'application des droits fondamentaux pour les étrangers, les médias font quelques articles d'estime alors que ce rapport devrait être un instrument de pédagogie et qu'il devrait être diffusé très largement. Lorsque les préfectures bloquent les files d'attente d'étrangers, il y a une attitude discriminatoire contraire à l'application des règles applicables à cette population comme à toute autre personne. Aujourd'hui, on se heurte à un discours unique et à un agenda des programmes politiques qui donnent la priorité à l'identité. Cette situation a été réactivée par les événements tragiques de 2015 et 2016 mais son origine se situe bien en amont, il y a dix à quinze ans déjà.

\section{$H \& M$ : Le débat sur les statistiques ethniques en France est-il définitivement abandonné ? Quels sont les critères à retenir pour mettre en place un matériel de mesure statistique des discriminations?}

J. T. : Il faut bien comprendre que l'on crée de la confusion à partir de l'expression «statistiques ethniques ». Ce qui est interdit et doit le demeurer, ce sont des fichiers nominatifs fondés sur certaines caractéristiques, en l'occurrence ethniques. Mais, en revanche, que l'on tienne compte d'un certain nombre de caractéristiques dans les études et les recherches scientifiques, ce n'est pas la même chose et c'est admis. Nous y sommes favorables, car cela permet de mesurer les itinéraires des personnes susceptibles d'être victimes de discrimination. Le débat sur les statistiques ethniques a démarré sur une mauvaise base parce qu'on confond l'utilisation économique des statistiques ethniques et les travaux qui étudient les réalités, et font évoluer les attitudes et les lois. Nous avons fait des propositions pour que soient développés dans les entreprises des indicateurs permettant d'évaluer les dispositifs qui favorisent la diversité dans les embauches, les parcours professionnels ou la lutte contre les discriminations : il faut tendre aux responsables des ressources humaines un miroir dans lequel ils puissent voir en permanence ce qu'ils ont fait ou non dans ce domaine. Cela permet de perfectionner les entreprises ou la fonction publique dans la mise en place de règles et dans la lutte contre les discriminations. C'est donc une fausse querelle que François Héran avait très bien analysée à la suite des travaux de la commission dont il avait été chargé. Depuis 2006, date du début de l'enquête Trajectoires et origines, nous mesurons les itinéraires des populations migrantes ou descendantes de migrants dans le cadre d'études scientifiques conduites, notamment, par l'Ined.

$H \& M$ : Les discriminations dans l'accès aux services publics et dans les relations avec l'administration, questionnent l'éthique et les attitudes professionnelles des agents face à certains publics. La police n'est-elle pas particulièrement ciblée ? Des formations sur les compréhensions des processus de discrimination sont-elles envisagées?

J. T. : Du point de vue du service public et de l'attitude des fonctionnaires, il y a trois questionnements. La question de la neutralité du service public et des agents publics est réglée par le principe de laïcité. Deuxièmement, sur l'égalité entre les agents du service public, une étude menée l’année dernière avec le ministère de la Fonction publique démontre que, malgré l'anonymat, malgré les grilles et les règlements égalitaires, il existe encore des différences de salaires et de traitement en moyenne de $12 \%$ entre les femmes et les hommes. Enfin, le troisième élément de discrimination relève des attitudes des agents du service public à l'égard de certains publics. Le Défenseur les a dénoncées, à la 
suite des positions de la Halde sur ce même thème. Ces réclamations concernent les cantines, les hôpitaux, la police, etc. Mais, de manière générale, nous arrivons à traiter ces situations avec l'utilisation des critères de la loi, en cherchant à ce que la fonction publique soit traitée comme le secteur privé. Il ne s'agit pas de protéger le service public, ni de le stigmatiser comme étant particulièrement discriminateur. Il faut faire des efforts, et c'est pour cela que nous avons besoin d'avoir un discours contraire à celui généralement répandu. C'est pour cela que je me suis réjoui que le Comité interministériel en avril 2015 ait abouti à un projet de loi Égalité et citoyenneté dans laquelle on ose enfin depuis dix ans réaffirmer la volonté de lutter contre les discriminations. Par ailleurs, dans à la loi Justice 21, le gouvernement a proposé une action collective contre les discriminations. Il ne peut pas y avoir de comportements exemplaires dans la fonction publique si, par ailleurs, le discours public et le climat général considèrent ces questions comme négligeables ou secondaires par rapport aux questions d'identité ou de sécurité. Le pôle santé du Défenseur des Droits montre, par exemple, que les cas de patientes dans les hôpitaux qui refusent d'être auscultées par des médecins hommes sont très rares. Les journaux partent souvent d'un cas pour amplifier le phénomène. Et souvent les cas se règlent tout seuls, sans besoin de médiatisation excessive.

Le problème est qu'aujourd'hui toutes ces questions sont instrumentalisées sur tous les fronts alors que les voies d'un règlement juridique sont toujours possibles. Exemple de l'indemnisation des dégâts occasionnés lors des perquisitions administratives liées à l'état d'urgence : le Conseil d'État demande que les procédures soient simplifiées et que, conformément à nos recommandations, la faute lourde de l'État ne soit pas exigée. Il y avait dans cette affaire une discrimination implicite puisque toutes les perquisitions ont été faites dans certains quartiers et auprès de certaines populations. Les délations ont stigmatisé des populations de confession musulmane. Pour régler ces situations très sensibles, il faut trouver des voies de droit en échappant au jeu de la politique.

En ce qui concerne les catégories d'agents publics visées par les attitudes discriminatoires, la police figure en bonne place parce qu'elle dispose de la violence légitime pour utiliser la contrainte dans l'application des lois. Mais la situation est asymétrique entre les forces de l'ordre et l'individu interpellé qui, lui, est à mains nues. Il faut donc appliquer des règles de contrôle et de restriction en faisant respecter la déontologie de la sécurité, ce dont le Défenseur des Droits est chargé. J'ai, par exemple, demandé que les contrôles des papiers d'identité aient une existence juridique et obéissent uniquement à des raisons objectives. La Cour d'appel de Paris en juin 2015 nous a donné raison ; la Cour de cassation va se prononcer bientôt. Nous proposons aussi des formations systématiques des gardiens de la paix, des commissaires de police, afin de les sensibiliser à la lutte contre les discriminations. En effet, ils sont souvent les premiers en contact avec les personnes ayant des difficultés ou en situation de conflit. Il faut donc que ces agents puissent acquérir une culture juridique dans ce domaine.

\section{$H \& M$ : Les associations de défense des droits humains et de lutte contre les discriminations, à l'échelle locale comme internationale, sont- elles des partenaires du Défenseur des Droits? Quel type de collaborations est mis en place? Le secteur associatif est-il suffisamment sou- tenu par les pouvoirs publics?}

J. T. : L'ancien Défenseur des Droits, Dominique Baudis, avait déjà repris un certain nombre de collaborations qui existaient du temps de la Halde. J'ai poursuivi et développé un système de partenariat. Dans la loi, sont prévus trois collèges consultatifs que je réunis cinq fois par an pour leur soumettre des projets et des initiatives, en particulier lorsqu'il s'agit de questions nouvelles. D'autre part, je réunis deux fois par an chacun des neuf comités d'entente et de liaison composés du Défenseur, de ses équipes et d'une douzaine d'associations représentatives au 
plan national, par exemple, les LGBT, le logement, les femmes, les handicapés, etc. Ces comités me permettent d'avoir un dialogue permanent avec les associations et la société civile : j'expose nos activités et nos projets, les associations me font remonter les préoccupations du terrain. Par exemple, j'ai eu l'idée de faire un rapport sur les droits fondamentaux des étrangers et la manière dont ils sont mal appliqués en France à la suite d'une réunion du comité d'entente femmes où le Planning familial a exposé les obstacles mis devant les femmes étrangères qui veulent recourir à une IVG. À travers les rapports, les observations adressées aux tribunaux et les notes, le Défenseur émet des jugements juridiques qui sont difficilement réfragables en matière de discriminations, ce qui implique une expertise sur chaque sujet. Par mes relations avec la société civile, je ne travaille pas « en chambre » mais sur une réalité sociale qui s'exprime bien entendu à la fois par les 100000 réclamations déposées chaque année, par tous ces partenariats avec des associations et des ONG, et par nos études et nos recherches. Les associations trouvent parfois chez le Défenseur l'écoute qu'elles n'ont pas auprès des pouvoirs publics par un soutien à leurs revendications en tant que minorités ayant des droits à défendre.

H\&M : Quelle politique publique concentre aujourd'hui l'essentiel des interventions de l'État en matière de lutte contre des discriminations? Que pensez-vous de cette " territorialisation " de la politique de lutte contre les discriminations ? Ne se fait-elle pas au détriment d'interventions auprès de certaines populations discriminées?

J. T. : Dans beaucoup de domaines (droits des enfants, des personnes âgées, des personnes handicapées), le Défenseur se retrouve face à un interlocuteur qui n'est plus l'État mais les départements, désormais compétents pour les solidarités. En particulier pour la protection sociale de l'enfance qui doit prendre en charge les mineurs étrangers isolés, nous avons à faire en quelque sorte à une cen- taine de politiques différentes. Les villes ont plutôt des compétences dans le domaine de l'éducation. C'est la même situation avec les Maisons départementales des personnes handicapées. Le Défenseur tente d'introduire un pilotage national, et l'idée de labéliser les bonnes pratiques des départements ou des villes serait efficace en termes de communication, même si certaines collectivités territoriales se font une gloire électorale de discriminer certaines populations, notamment les populations roms. Quand des communes n'inscrivent pas les enfants roms dans les écoles ou les mettent dans des écoles séparées, les maires se retournent vers leurs administrés pour vanter le fait que leur politique les protège contre les étrangers. Il faut faire très attention, dans l'ambiance politique actuelle, aux discours identitaires. Je privilégie donc les actions continues avec les associations des maires de France, des départements de France, des grandes villes, pour travailler avec elles sur de nombreux sujets, y compris la lutte contre les propos racistes, antisémites, xénophobes et homophobes sur les réseaux sociaux, via le site «Égalité contre le racisme » qui a été créé l'année dernière pour servir de portail des initiatives et des ressources pédagogiques. Ceci dit, il n'y a pas vraiment de géographie des territoires qui seraient plus discriminatoires que d'autres : certains départements ruraux sont très accueillants, d'autres très refermés sur eux-mêmes. Certaines villes sont ouvertes et communiquent beaucoup sur la diversité culturelle des populations qui y résident et d'autres y sont hostiles. Pour la situation des mineurs isolés étrangers, on sait très bien que leur situation est difficile dans les départements du Nord, de la Seine-Saint-Denis, de Paris et dans les Bouches-du-Rhône, malgré les efforts consentis par ces départements. On peut pas dire non plus que les villes françaises changent d'attitude en fonction de leur couleur politique. Ce sont les dépenses de solidarité qui sont les plus flexibles dans leur budget et sont quelquefois sacrifiées en fonction de la demande de l'opinion locale et des contextes budgétaires de plus en plus serrés. 